\title{
Polinização por vibração e sistema reprodutivo de duas espécies de Sauvagesia L. (Ochnaceae)
}

\author{
TARCILA DE LIMA NADIA ${ }^{1}$ e ISABEL CRISTINA MACHADO ${ }^{1,2}$
}

(recebido: 29 de janeiro de 2004; aceito: 9 de dezembro de 2004)

\begin{abstract}
Buzz pollination and breeding system of two species of Sauvagesia L. (Ochnaceae)). Reproductive phenology, floral biology, pollination, and breeding system of Sauvagesia erecta L. and S. sprengelii A. St.-Hil. were studied, respectively, in a remnant of Atlantic forest, located at the "Parque Estadual Dois Irmãos", Recife, and in open areas, Goiana, both in Pernambuco state, northeastern Brazil. The species have pollen flowers with poricidal anthers and a staminodial envelope, which surrounds the stamens and the gynoecium, creating an apical pore to pollen release. The pattern of flowering of both species is continual. The species are self-compatible, autogamic and do not set apomictic fruits. Exclusively bees, mainly of the families Apidae and Halictidae, visited the flowers of both species. Bees of the genus Paratetrapedia visited only $S$. erecta flowers. Bombus brevivillus Franklin, Florilegus similis Urban, Xylocopa muscaria Fabricius were observed visiting only $S$. sprengelii flowers. Nevertheless, Augochloropsis species visited flowers of both species. Buzz pollination mechanism takes place with all these bee species acting as effective pollinators, excepting for one species of Paratetrapedia, which is a pollen thief. According to the pollinators' behaviour, the staminodial envelope has a function on the buzz pollination process, which characterizes a mechanism of transference of function, already mentioned to the family.
\end{abstract}

Key words - Atlantic Forest, buzz pollination, melitthophily, Ochnaceae, Sauvagesia

RESUMO - (Polinização por vibração e sistema reprodutivo de duas espécies de Sauvagesia L. (Ochnaceae)). Foram estudados a fenologia, a morfologia floral, o sistema de polinização e de reprodução de Sauvagesia erecta L. e S. sprengelii A. St.-Hil., respectivamente, em um remanescente de Mata Atlântica, localizado no Parque Estadual Dois Irmãos, Recife, e em áreas abertas, em Goiana, litoral norte de Pernambuco. As espécies apresentam flores de pólen com anteras poricidas envolvidas por um cone, formado por estaminódios petalóides, deixando apenas um poro apical, através do qual o pólen é liberado. As espécies possuem um padrão de floração contínuo. Ambas são auto-compatíveis, autógamas e não formam frutos apomíticos. Foram observadas visitas de abelhas das famílias Apidae e Halictidae, as quais polinizam as flores através da vibração das anteras. Duas espécies de Paratetrapedia visitaram apenas flores de S. erecta. Bombus brevivillus Franklin, Florilegus similis Urban e Xylocopa muscaria Fabricius foram observadas somente em flores de $S$. sprengelii. Por sua vez, as espécies de Augochloropsis visitaram flores de ambas as espécies. Todas essas abelhas vibram o cone de estaminódios e as anteras e podem polinizar a flor, com exceção de uma espécie de Paratetrapedia. De acordo com o comportamento dos visitantes, observa-se que o cone de estaminódios tem uma função na polinização por vibração, equivalente a uma antera poricida, tratando-se, portanto, de uma transferência de função, já mencionada anteriormente para a família.

Palavras-chave - Mata Atlântica, melitofilia, Ochnaceae, polinização por vibração, Sauvagesia

\section{Introdução}

A polinização por vibração ("buzz-pollination", sensu Buchmann 1983) ocorre geralmente em flores com anteras poricidas e que apresentam pólen como único recurso floral, as chamadas flores de pólen (Vogel 1978). Para o pólen ser expelido das anteras poricidas é necessário que o animal visitante, exclusivamente determinadas abelhas fêmeas, efetue vibrações que são originadas da contração e relaxamento dos músculos de vôo (Buchmann 1983).

\footnotetext{
1. Universidade Federal de Pernambuco, Departamento de Botânica, 50372-970 Recife, PE, Brasil.

2. Autor para correspondência: imachado@ufpe.br
}

Há três tipos de abelhas que visitam flores com esse tipo de mecanismo de polinização. As vibradoras, representadas pelas abelhas que realmente transmitem vibrações para as anteras, para poder coletar pólen; as coletoras, representadas por aquelas abelhas que recolhem o pólen que se encontra na superfície da flor após esta ter sido vibrada; as mordedoras, representadas por abelhas que danificam as anteras, mastigando-as, para coletar o pólen (Wille 1963). Os dois últimos tipos geralmente não efetuam a polinização, apenas pilham pólen; por sua vez, as abelhas mordedoras, além de não polinizarem, também danificam os estames das flores. Isso ocorre freqüentemente em espécies de Melastomataceae, que apresentam o mecanismo de polinização por vibração (Renner 1983, 1989).

Polinização por vibração tem ampla distribuição nas Angiospermas, ocorrendo em diferentes famílias 
(Buchmann 1983). A família Ochnaceae possui cerca de 28 gêneros e 400 espécies tropicais e subtropicais (Barroso 1978) e pode ser dividida em duas subfamílias, Ochnoideae e Sauvagesioideae (Amaral \& Bittrich 1998). Espécies do gênero Sauvagesia L., incluído na subfamília Sauvagesioideae, apresentam mecanismo de polinização por vibração. Os estames dessas espécies podem ou não apresentar anteras poricidas, e estão envolvidos por estaminódios petalóides dispostos de forma a deixar apenas um orifício apical para a liberação do pólen (Kubitzki \& Amaral 1991). Devido a essa organização, além da função de proteção, Kubitzki \& Amaral (1991) atribuíram aos estaminódios petalóides função no processo de polinização vibrátil, devido ao poro apical formado por eles ao invés de propriamente a presença de anteras com deiscência poricida. Esse fenômeno, em que a função de um determinado órgão é transferida para um outro, foi denominado por Corner (1958) de transferência de função.

Estudos sobre polinização na família Ochnaceae foram realizados por Kubitzki \& Amaral (1991), que enfatizaram a ocorrência de transferência de função no sistema de polinização da família, por Pinheiro et al. (1999), que estudaram a ecologia da polinização, o sistema reprodutivo e a sequiência de antese de Ouratea cuspidata (A. St.-Hil.) Engler e por Henriques (1999), que abordou aspectos sobre a ecologia da polinização de Ouratea hexasperma (A. St.-Hil.) Baill. Além desses, Amaral \& Bittrich (1998) desenvolveram um trabalho sobre a origem dos estaminódios petalóides para a subfamília Sauvagesioidae.

Este trabalho compara os sistemas de reprodução e de polinização de duas espécies de Sauvagesia ( $S$. erecta L. e S. sprengelii A. St.-Hil.), objetivando analisar as interações e adaptações existentes entre as plantas e seus polinizadores e verificar a função do cone de estaminódios petalóides para ambas as espécies.

\section{Material e métodos}

O estudo com Sauvagesia erecta L. foi realizado no Parque Estadual Dois Irmãos, um remanescente de Mata Atlântica urbana, localizado no município de Recife,

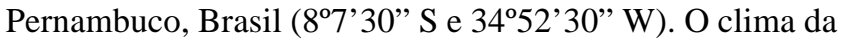
região é tropical costeiro, quente (temperatura mínima de $18^{\circ} \mathrm{C}$ ) e úmido, apresentando estação seca no período de outubro a janeiro e estação úmida de março a agosto, com precipitação média anual total de aproximadamente $2.460 \mathrm{~mm}$ e temperaturas médias mensais superiores a $23{ }^{\circ} \mathrm{C}$ (Coutinho et al. 1998). Duas populações de Sauvagesia erecta foram estudadas. Uma localizada às margens do Açude do Meio e outra, próximo ao Açude do Prata, em áreas periodicamente alagadas, apresentando cerca de $22,5 \mathrm{~m}^{2}$ e $60 \mathrm{~m}^{2}$, respectivamente. Em ambas as populações, os indivíduos situavam-se muito próximos entre si e apresentavam muitas ramificações.

Sauvagesia sprengelii $\mathrm{A}$. St.-Hil. foi estudada em áreas abertas, próximas a manguezais localizados na Praia de Catuama, Município de Goiana, Pernambuco, Brasil (7 $7^{\circ} 33^{\prime}$ S e $\left.35^{\circ} 00^{\prime} \mathrm{W}\right)$. A região apresenta precipitação média anual de $2.053 \mathrm{~mm}$, com temperaturas máximas e mínimas mensais médias de $29,9^{\circ} \mathrm{C}$ e $21,9^{\circ} \mathrm{C}$, respectivamente (fonte: ITEP). $\mathrm{O}$ período seco compreende os meses de setembro a janeiro, com precipitação média mensal menor que $100 \mathrm{~mm}$, e o período úmido, de fevereiro a agosto, com precipitação mensal podendo alcançar $319 \mathrm{~mm}$ no mês de julho. Indivíduos de Sauvagesia sprengelii distanciavam-se cerca de $30 \mathrm{~cm}$ uns dos outros e as duas populações estudadas tinham uma área total aproximada de $100 \mathrm{~m}^{2}$ e $360 \mathrm{~m}^{2}$.

$\mathrm{O}$ estudo da fenologia reprodutiva foi feito no período de setembro de 2001 a agosto de 2002 para Sauvagesia erecta e de maio de 2002 a abril de 2003 para S. sprengelii. Foi marcado um total de 10 indivíduos nas duas populações para cada espécie, sendo contados, quinzenalmente, o número de botões, flores e frutos, obtendo-se, assim, a intensidade de floração e frutificação para cada indivíduo (Bencke \& Morellato 2002). Desse modo, o pico dessas fenofases foi determinado baseado no mês que apresentou o maior número de indivíduos com maior intensidade de floração e frutificação. Em ambas as espécies, outros indivíduos ocorrentes nas populações também foram observados quanto à presença ou ausência de flores. Os padrões fenológicos de S. erecta e $S$. sprengelii foram determinados a partir dos modelos de Gentry (1974) e Newstrom et al. (1994).

Foram utilizadas 10 flores ainda não visitadas, de cada espécie, para análise e descrição de sua morfologia. Nessas flores, foram contados os números de sépalas, pétalas, estaminódios filiformes e petalóides, estames, carpelos e óvulos; medidos os comprimentos dos estaminódios filiformes e petalóides, estames e estilete, sendo observado o tipo de deiscência das anteras. No campo, foram medidos, com régua milimetrada, os diâmetros do cálice e da corola. Foram feitos desenhos esquemáticos das estruturas florais em câmara clara acoplada a estereomicroscópio. O início, duração e final da antese foram verificados em campo. A receptividade do estigma foi analisada seguindo-se a metodologia de Robinsohn (1924). O número de grãos de pólen/flor foi estimado com auxílio de câmara de Neubauer (Maêda 1985), sendo estimada a razão P/O, segundo a metodologia de Cruden (1977). A viabilidade polínica foi estimada por meio da coloração do citoplasma pelo carmim acético (Radford et al. 1974). Para cada experimento foram coletados 10 botões florais de indivíduos diferentes e utilizadas duas anteras de cada botão, totalizando 20 anteras para cada espécie. Espécimes-testemunho foram depositados no Herbário-UFP, Departamento de Botânica, Universidade Federal de Pernambuco, sob os números 29.780(1), 31.123(8), 30.334(2) e 31.124(6). 
Para o estudo do sistema reprodutivo de ambas as espécies foram ensacados botões de indivíduos diferentes e, à medida em que as flores iam abrindo, eram realizados os testes de autopolinização espontânea (apenas marcando a flor que se encontrava aberta e reensacando para verificar a formação de fruto sem manipulação) e autopolinização manual (colocando o pólen da própria flor em seu estigma). A polinização cruzada foi feita em flores previamente emasculadas e ensacadas. Para verificar a formação natural de frutos, foram marcadas flores de indivíduos diferentes. Para verificar a ocorrência de apomixia foram emasculados 10 botões em pré-antese de indivíduos diferentes de cada espécie, ensacando-os até a formação ou não de frutos. Em Sauvagesia erecta, observou-se, ainda, a eficiência do polinizador, sendo emasculados e não ensacados 30 botões de indivíduos diferentes.

O comportamento dos visitantes florais foi analisado através de observações diretas no campo em um total de 284 horas, sendo 140 horas para $S$. erecta e 144 horas para S. sprengelii. A freqüência de visitas foi calculada em um período de nove horas consecutivas para cada espécie, sendo considerada uma visita o ato da abelha pousar na flor e realizar movimentos vibratórios. Os insetos que visitaram as flores de $S$. erecta e $S$. sprengelii foram coletados com frascos de vidro contendo acetato de etila. Posteriormente, foram montados a seco, enviados para especialistas para identificação e depositados na coleção de insetos polinizadores do Laboratório de Biologia Floral e Reprodutiva da UFPE. A disponibilidade polínica para ambas as espécies foi verificada a partir da contagem da quantidade de grãos de pólen por flor, com auxílio de câmara de Neubauer (Maêda 1985), em diferentes flores a cada duas horas, após o início das visitas (6:00 h) até o seu término (12:00 h).

Foram aplicados o teste $\mathrm{t}$, para verificar diferenças de distribuição normal entre as estruturas florais de $S$. erecta e S. sprengelii, o teste de Mann-Whitney, para analisar diferença entre as estruturas florais de distribuição não normal, os testes $\mathrm{F}^{2}$ e $\mathrm{G}$, para avaliar diferenças na quantidade de pólen viáveis entre as espécies e no número de frutos formados entre os tratamentos do sistema reprodutivo para ambas as espécies, respectivamente, e ANOVA, para verificar diferença no número de sementes entre os tratamentos de cada espécie. Os testes foram feitos com o auxílio do software BioEstat 2.0 (Ayres et al. 2000).

\section{Resultados e Discussão}

Sauvagesia erecta L. é uma erva que apresenta padrão de floração e de frutificação do tipo contínuo, segundo a classificação de Newstrom et al. (1994). Os picos de floração e de frutificação variaram de indivíduo para indivíduo, sendo que o maior número de indivíduos apresentou um pico de floração em janeiro e de frutificação em março (figura 1A). Esse último pode ser o resultado do pico de floração observado em janeiro. $\mathrm{O}$ número de flores produzidas por indivíduo é baixo (figura 1B), caracterizando o tipo estado constante ("steady state"), segundo classificação de Gentry (1974). Sauvagesia sprengelii A. St.-Hil. é um subarbusto anual, com cerca de $20 \mathrm{~cm}$ de altura. Em nível individual, a floração é anual, porém, em nível de população, a floração é contínua, uma vez que durante todo ano há indivíduos em floração. Analisando-se exclusivamente os 10 indivíduos marcados, estes apresentaram dois picos de floração e frutificação nos meses de maio e outubro, produzindo poucas flores por indivíduo, e ausência de flores e frutos de dezembro a abril (figura 2A-B), indicando uma floração anual (sensu Newstron et al. 1994).

O padrão contínuo de floração também foi observado por outros autores que estudaram espécies
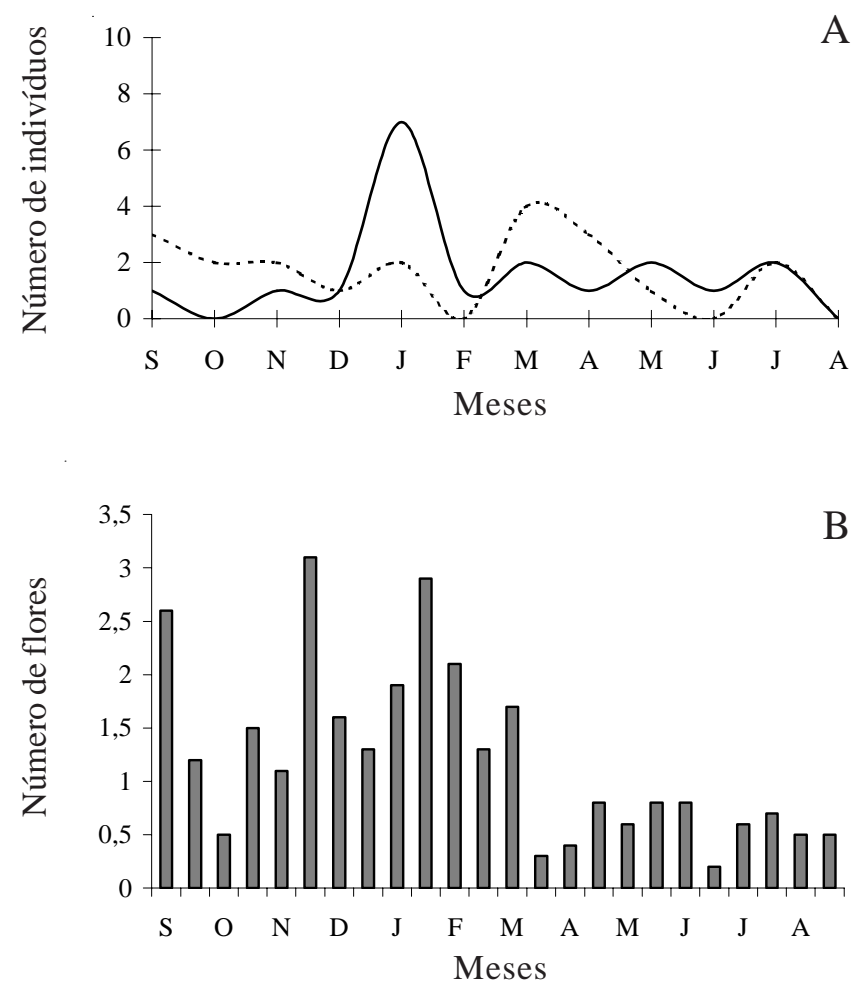

Figura 1. Número de indivíduos em pico de floração e frutificação (A) e número de flores abertas por indivíduo (B) no período de setembro/2001 a agosto/2002 para Sauvagesia erecta. - Floração; $\cdots$ = Frutificação $;=$ = Número de flores abertas.

Figure 1. Number of individuals in flowering and fruiting peak (A) and number of opened flowers per individual (B) in the period of September/2001 to August/2002 of Sauvagesia erecta.$-=$ Flowering; $\cdots=$ Fruiting $;=$ = Number of opened flowers. 

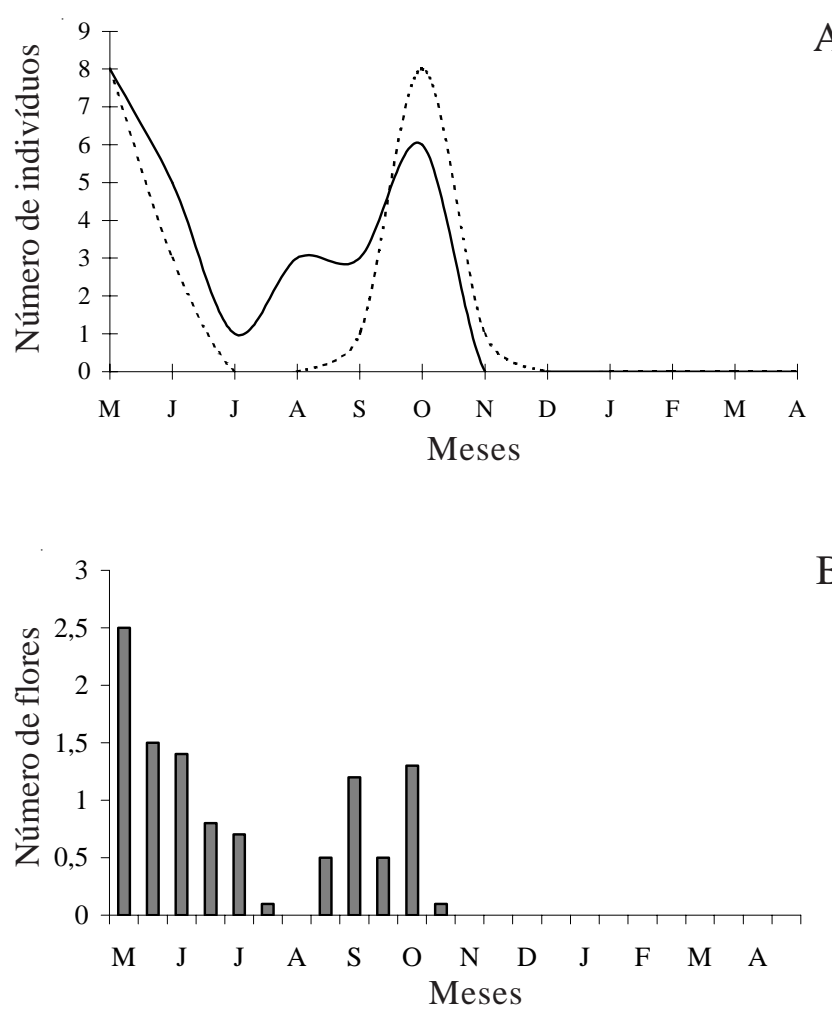

Figura 2. Número de indivíduos em pico de floração e frutificação (A) e número de flores abertas por indivíduo (B) no período de maio/2002 a abril/2003 para Sauvagesia sprengelii. $-=$ Floração; $\cdots \cdot=$ Frutificação; $\square=$ =Número de flores abertas.

Figure 2. Number of individuals in flowering and fruiting peak (A) and number of opened flowers per individual (B) in the period of May/2002 to April/2003 of Sauvagesia sprengelii. $-=$ Flowering; $\cdots .=$ Fruiting; $\square=$ Number of opened flowers.

com flores de pólen, como Buchmann et al. (1978) em Solanum douglasii Dunal e Solanum xanti Gray; Gottsberger \& Silberbauer-Gottsberger (1988) em Chamaecrista campestris Irwin \& Barneby, C. flexuosa (L.) Greene e C. cathartica (Mart.) Irwin \& Barneby var. cathartica; Oliveira Filho \& Oliveira (1988) em Solanum lycocarpum A. St.-Hil.; Melo \& Machado (1996, 1998) em Henriettea succosa DC. e Miconia ciliata (L.C. Rich.) DC.; Pinheiro et al. (1999) em Ouratea cuspidata (A. St.-Hil.) Engler; Bezerra \& Machado (2003) em Solanum stramonifolium Jacq. Esse padrão permite a disponibilidade de recursos para seus polinizadores durante todo o ano.

As flores de $S$. erecta são pêndulas e estão dispostas três a três na axila foliar, diferindo das outras espécies da família Ochnaceae, que normalmente apresentam flores em panículas terminais (Barroso 1978), como ocorre em S. sprengelii. As flores de ambas as espécies são actinomorfas, possuem cinco sépalas verdes e cinco pétalas brancas, dispostas alternadamente entre si (figuras 3, 4, 5). $\mathrm{O}$ androceu nas duas espécies é composto por uma série (tabela 1) de estaminódios filiformes, cinco estaminódios petalóides e cinco estames (figuras 3, 4, 5). Os estames possuem um curto filete e suas anteras amarelo-pálidas apresentam uma fenda (figura $3 \mathrm{C}, \mathrm{F}$ ) que se estende do ápice até um terço $(S$. erecta) ou um quarto (S. sprengelii) do seu comprimento, ainda sendo classificadas como anteras poricidas, considerando definição de Buchmann (1983). Os estaminódios petalóides de cor lilás envolvem os estames e o gineceu, deixando apenas um poro apical, e os estaminódios filiformes também lilases circundam os petalóides (figura 3A, B, D, E). Desse modo, observa-se uma visível diferença na coloração entre as pétalas e os estaminódios, como ocorre em flores de Ochnaceae que possuem estaminódios petalóides envolvendo os estames (Kubitzki \& Amaral 1991).

As flores de $S$. erecta e $S$. sprengelii oferecem pólen como única recompensa floral e podem ser classificadas como flores de pólen do tipo Solanum (sensu Vogel 1978) por apresentarem poucos estames com anteras alongadas e poricidas. Segundo Vogel (1978), flores de pólen devem conter anteras vistosas que sinalizem, para as abelhas, fonte de alimento (pólen). Em $S$. erecta e $S$. sprengelii, porém, as anteras, além de apresentarem cores pálidas, não são visíveis externamente, uma vez que estão encobertas pelos estaminódios. Dessa forma, o contraste de cores entre as pétalas e os estaminódios nestas duas espécies de Sauvagesia provavelmente desempenha esta função de atração visual que geralmente é exercida pelas anteras em outras espécies cujo recurso é exclusivamente pólen, como mencionado por Vogel (1978). De maneira semelhante, espécies de Conostephium (Epacridaceae), polinizadas por vibração, apresentam anteras escondidas dentro do tubo da corola, sendo o contraste de cores observado entre a porção exposta da corola e o conjunto de sépalas e brácteas (Houston \& Ladd 2002).

$\mathrm{O}$ gineceu de ambas espécies é formado por um ovário tricarpelar, unilocular, que se encontra sobre um curto ginóforo. As flores de $S$. sprengelii são significativamente menores que as de $S$. erecta, possuindo menor número de estaminódios filiformes, óvulos e grãos de pólen/flor (tabela 1). No entanto, a razão $\mathrm{P} / \mathrm{O}$ de ambas as espécies não difere significativamente. Sauvagesia erecta e $S$. sprengelii apresentaram elevada razão P/O (sensu Cruden 2000) como ocorre geralmente em espécies que oferecem 
pólen como única recompensa floral (Cruden 2000). Cruden $(1997,2000)$ também relaciona a alta razão pólen/ óvulo às espécies xenógamas obrigatórias. No entanto, S. erecta e $S$. sprengelli são autogâmicas. Desse modo, o fato das espécies apresentarem pólen como único recurso floral pode ser o determinante mais importante da alta razão $\mathrm{P} / \mathrm{O}$, já que o pólen é utilizado tanto pela planta (na polinização e fecundação) quanto pelo polinizador (para alimento).

A antese em $S$. erecta e $S$. sprengelii inicia em torno das 5:00 h, com a abertura gradativa dos botões, estando as flores completamente abertas às 6:00 h. O estigma se encontra receptivo desde o início da antese em ambas as espécies. As flores de $S$. erecta duram aproximadamente oito horas, com a senescência das pétalas iniciando às 13:00 h, enquanto as de $S$. sprengelii têm uma duração de seis horas, uma vez que as pétalas começam a murchar em torno das 11:00 h. Flores com curta longevidade são comuns em espécies que apresentam flores pequenas (Primack 1985, Stratton 1989), podendo também estar relacionadas ao fato das espécies se autopolinizarem (Primack 1985).

Os resultados dos experimentos envolvendo o sistema reprodutivo estão sumarizados na tabela 2 . Sauvagesia erecta e $S$. sprengelii produzem frutos tanto na autopolinização espontânea quanto na manual, não sendo observados frutos apomíticos. A produção de frutos espontaneamente garante a propagação das espécies em períodos com baixa freqüência de polinizadores.

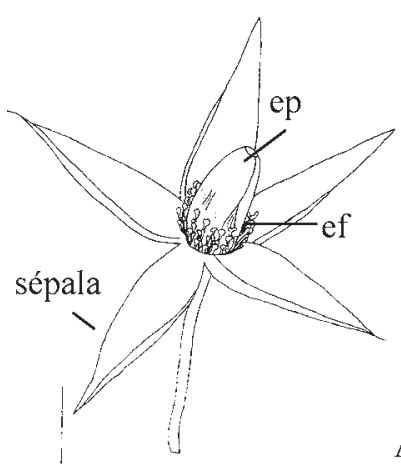

A
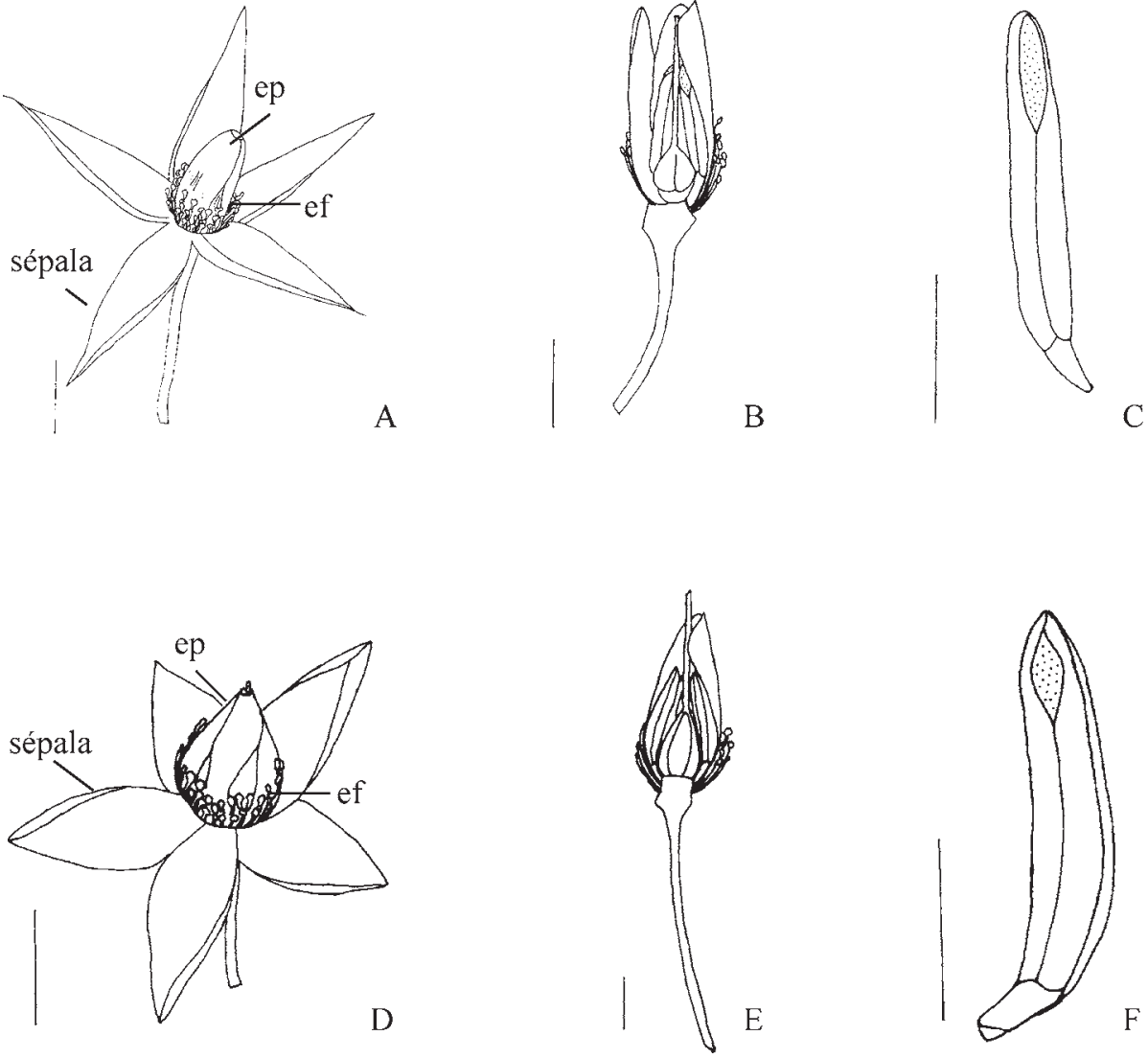

B

$\mathrm{C}$

Figura 3. Esquemas das estruturas florais de Sauvagesia erecta (A-C) e Sauvagesia sprengelii (D-F). A, D. Organização dos estaminódios filiformes (ef) e petalóides (ep). B, E. Corte longitudinal do cone de estaminódios, mostrando a disposição do gineceu e das anteras. C, F. Antera evidenciando o poro apical. Para maior clareza alguns elementos florais foram retirados. Barras $=2 \mathrm{~mm}(\mathrm{~A}, \mathrm{~B}, \mathrm{D}) ; 1 \mathrm{~mm}(\mathrm{C}, \mathrm{E}, \mathrm{F})$.

Figure 3. Schemes of floral structure of Sauvagesia erecta (A-C) and Sauvagesia sprengelii (D-F). A, D. Organization of filiform (ef) and petaloid (ep) staminodia. B, E. Longitudinal section of staminodial cone, showing the arrangement of gynoecium and anthers. C, F. Anther evidencing the apical pore. For better understanding some floral parts were removed. Bars $=2 \mathrm{~mm}$ $(\mathrm{A}, \mathrm{B}, \mathrm{D}) ; 1 \mathrm{~mm}(\mathrm{C}, \mathrm{E}, \mathrm{F})$. 
A formação de frutos em $S$. erecta foi maior na autopolinização manual que nos outros tratamentos (tabela 2), o que comprova a auto-compatibilidade da espécie, podendo também indicar baixa eficiência dos polinizadores e maior investimento na autogamia pela planta. O teste de eficiência do polinizador reforça essa idéia (tabela 2). Wendt et al. (2002) também relacionaram o alto índice de autogamia de Pitcairnia corcovadensis Wawra (Bromeliaceae) à baixa eficiência do polinizador. Não houve diferença significativa no número de frutos e sementes formados entre os tratamentos.

Sauvagesia sprengelii, embora também sendo autogâmica e auto-compatível, apresentou maior sucesso reprodutivo na formação natural de frutos $(\mathrm{g}=3,97$; graus de liberdade (g.l. $)=1 ; \mathrm{p}=0,0464 ; \mathrm{g}=4,29 ; \mathrm{g} .1 .=1$; $\mathrm{p}=0,0384 ; \mathrm{g}=5,24 ; \mathrm{g} .1 .=1 ; \mathrm{p}=0,0220$ ), enquanto a produção de frutos entre os outros tratamentos foi semelhante. $\mathrm{O}$ número de sementes formadas também foi maior na formação natural de frutos, porém não houve diferença estatística entre os tratamentos, exceto entre o controle e a autopolinização manual $(\mathrm{t}=2,54$; g.l. $=3$; $\mathrm{p}=0,0173$ ).

Comparando-se o sucesso reprodutivo entre as duas espécies, não houve diferença significativa na formação de frutos entre os tratamentos, exceto no experimento de autopolinização manual $(g=6,11 ; g .1 .=1 ; p=0,0134)$. O número de sementes formadas nos tratamentos para $S$. erecta aparentemente é superior ao número de sementes dos tratamentos para $S$. sprengelii, porém não há diferença na razão óvulo/semente entre as duas espécies, exceto na autopolinização manual $(g=4,69$; g.l. $=1 ; \mathrm{p}=0,0303)$. A baixa produção de frutos e sementes de $S$. sprengelii em relação a $S$. erecta no tratamento de autopolinização manual pode ser devido à maior eficiência do polinizador, visto que $S$. sprengelii apresentou maior sucesso reprodutivo na formação natural de frutos (tabela 2). Duas espécies de Pitcairnia estudadas por Wendt et al. (2002) também apresentaram maior produção de frutos na autopolinização manual em relação à polinização cruzada, como ocorreu com Sauvagesia erecta; neste caso, a autogamia das espécies foi interpretada como sendo um mecanismo de isolamento reprodutivo por serem espécies congenéricas e simpátricas. No caso de $S$. erecta e S. sprengelii, essa interpretação não se aplica uma vez que suas populações estão geograficamente isoladas.

Com relação à viabilidade polínica, não houve diferença significativa entre as duas espécies: Sauvagesia erecta apresentou $91,89 \%$ de seus grãos de pólen viáveis e $S$. sprengelii, 98,22\%. Outras espécies que fornecem pólen como única recompensa floral também apresentaram alta viabilidade polínica (Buchmann et al. 1978, Oliveira Filho \& Oliveira 1988, Melo \& Machado 1996, 1998, Pinheiro et al. 1999, Henriques 1999). Segundo Richards (1986), espécies auto-incompatíveis apresentam alta viabilidade polínica. No entanto, Sauvagesia erecta e S. sprengelii são auto-compatíveis, fato que pode estar relacionado ao ambiente em que elas ocorrem, visto que plantas que habitam locais bastante perturbados tendem a ser autocompatíveis e/ou autógamas (Stebbins 1957).

Sauvagesia erecta é visitada por quatro espécies de abelhas, duas pertencentes à família Halictidae, gênero Augochloropsis, e duas da família Apidae, gênero Paratetrapedia (tabela 3). As espécies de Augochloropsis e uma espécie de Paratetrapedia possuem comportamento de vibração similar durante a coleta de pólen nas flores. Ao chegar na flor, a abelha pousa envolvendo o cone formado pelos estaminódios petalóides e realiza movimentos vibratórios (figura 6); nesse momento entra em contato com o estigma efetuando a polinização, pois este se localiza no mesmo nível do poro formado pelos estaminódios petalóides. Durante a vibração, o pólen é liberado, aderindo ao esterno do tórax da abelha. Durante a visita, as espécies de Augochloropsis apresentaram movimentos rotatórios em torno do androceu. Em alguns momentos, seguraramse com as pernas dianteiras no androceu, ficando suspensas, e com as pernas medianas transferiram o pólen do seu corpo para as pernas posteriores (figura 7). Tal comportamento, em que a abelha transfere o pólen para a escopa, foi observado por Michener et al. (1978) para espécies da família Halictidae.

A outra espécie de Paratetrapedia observada não apresenta comportamento de vibração, coletando os grãos de pólen que ficam por volta do poro formado pelos estaminódios petalóides, não efetuando a polinização. Essa abelha enquadra-se no tipo "coletor", seguindo terminologia de Wille (1963), pois não danifica a estrutura das anteras. A presença de abelhas mordedoras não foi registrada, estas, no entanto, são freqüentes em espécies de Melastomataceae (Renner 1983, 1989). Os estaminódios petalóides em Sauvagesia, portanto, provavelmente têm também uma função de proteção às anteras.

Seis espécies de abelhas visitam as flores de S. sprengelii: três da família Apidae (Bombus brevivillus Franklin, 1913, Florilegus similis Urban, 1970 e Xylocopa muscaria (Fabricius, 1778)) e três da família Halictidae, gênero Augochloropsis (tabela 3). Todas apresentam comportamento de vibração e são 
polinizadoras. As espécies de Augochloropsis apresentam comportamento similar ao descrito nas visitas a $S$. erecta. As outras espécies de abelhas apresentaram comportamento de vibração similar ao de Augochloropsis, não sendo observada transferência de pólen para as escopas.

Sauvagesia erecta e $S$. sprengelii partilham um único polinizador, Augochloropsis sp.1 (tabela 3), talvez pelas outras espécies não ocorrerem concomitantemente em ambos os locais. Martins (1995), em um levantamento da flora apícola na Chapada Diamantina,
Bahia, coletou 28 espécies de abelhas em flores de Sauvagesia sprengelii, inclusive machos. No entanto, segundo Buchmann (1983), apenas abelhas fêmeas visitam flores de pólen, uma vez que os machos não utilizam esse recurso.

Espécies de Bombus e Xylocopa, abelhas de médio e grande porte, são conhecidas como polinizadoras de várias espécies que apresentam mecanismo de polinização por vibração, tais como Cassia fasciculata Michx. (Thorp \& Estes 1975), Solanum elaeagnifolium Willd, S. dulcamara L., Dodecatheon meadia L.,
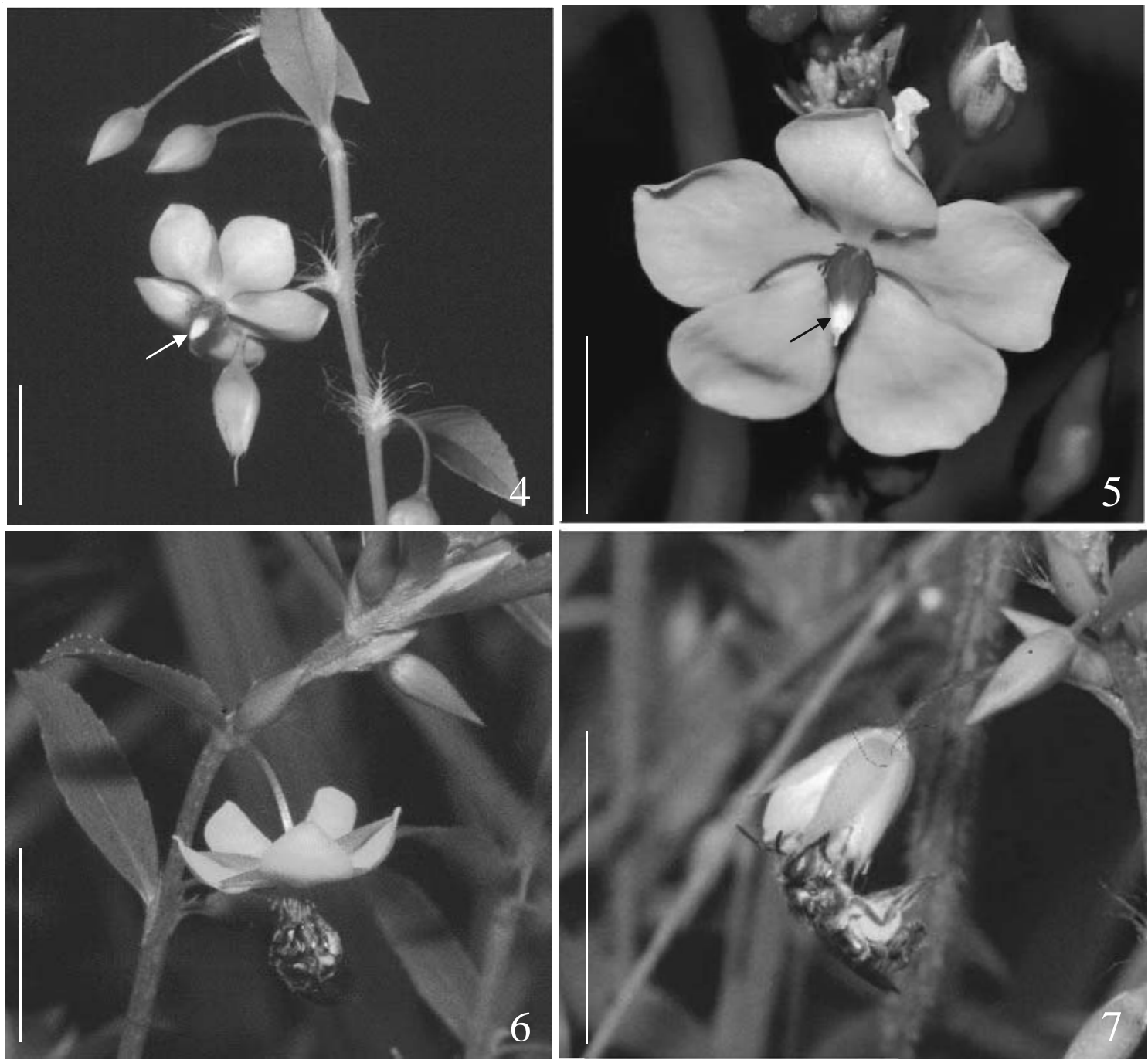

Figuras 4-7. Aspectos da morfologia floral e comportamento dos visitantes florais de Sauvagesia erecta e Sauvagesia sprengelii. 4-5. Flor de Sauvagesia erecta e Sauvagesia sprengelii, respectivamente. Notar cone de estaminódios no centro da flor (setas). 6. Augochloropsis sp. envolvendo o cone de estaminódios. 7. Augochloropsis sp. removendo o pólen de seu corpo com auxílio das pernas medianas. Barras $=1 \mathrm{~cm}$.

Figures 4-7. Aspects of the floral morphology and behaviour of the floral visitors of Sauvagesia erecta and Sauvagesia sprengelii. 4-5. Flower of Sauvagesia erecta and Sauvagesia sprengelii, respectively. Observe the staminodial cone in the center of flower (arrows). 6. Augochloropsis sp. involving the staminodial cone. 7. Augochloropsis sp. removing the pollen of its body with help of median legs. Bars $=1 \mathrm{~cm}$. 
Tabela 1. Quantidade e dimensões das estruturas florais, número de grãos de pólen por flor e razão P/O de Sauvagesia erecta e S. sprengelii $(\mathrm{g} .1 . \mathrm{l}=18 ; \mathrm{p}<0,001) . \mathrm{N}=10$. *Diâmetro, **Comprimento. $(\mathrm{t}=$ resultado do teste $\mathrm{t} ; \mathrm{u}=$ resultado do teste de Mann-Whitney).

Table 1. Quantity and dimensions of floral structure, number of pollen grains per flower and P/O rate of Sauvagesia erecta and S. sprengelii $(\mathrm{g} .1 .=18 ; \mathrm{p}<0.001) . \mathrm{N}=10 . *$ Diameter, ${ }^{*}$ Length. $(\mathrm{t}=\mathrm{t}$ test result; $\mathrm{u}=$ Mann-Whitney test result).

\begin{tabular}{lcccccc}
\hline \multirow{2}{*}{ Caracteres } & \multicolumn{2}{c}{ Sauvagesia erecta } & & \multicolumn{2}{c}{ Sauvagesia sprengelii } & \multirow{2}{*}{ Análise estatística } \\
\cline { 2 - 3 } & $\mathrm{N}$ & Dimensão $(\mathrm{mm})$ & $\mathrm{N}$ & Dimensão $(\mathrm{mm})$ & \\
\hline Cálice (sépalas) & 5 & $11,5( \pm 0,85)^{*}$ & & 5 & $6,1( \pm 0,88)^{*}$ & $\mathrm{u}=0,0$ \\
Corola (pétalas) & 5 & $15,8( \pm 0,92)^{*}$ & & 5 & $12,9( \pm 1,19)^{*}$ & $\mathrm{u}=2,5$ \\
Estaminódios Filiformes & $58( \pm 14,12)$ & $0,5,1 \mathrm{e} 1,5^{* *}$ & & $26,8( \pm 5,09)$ & $1 \mathrm{e} 1,5^{* *}$ & $\mathrm{t}=6,63$ \\
Óvulos & $56( \pm 6,40)$ & - & & $27,5( \pm 4,90)$ & - & $\mathrm{t}=11,34$ \\
N. de pólen/flor & $252.187,50( \pm 47.049,44)$ & - & & $106.375( \pm 41.959,18)$ & - & $\mathrm{u}=4,0$ \\
Razão P/O & $4.564,41( \pm 1.152,80)$ & - & $4.040,28( \pm 1.915,34)$ & - & - \\
\hline
\end{tabular}

Lycopersicon esculentum Mill. (Symon 1979), Cassia biflora L. (Wille 1963), Solanum lycocarpum (Oliveira Filho \& Oliveira 1988), Henriettea succosa (Melo \& Machado 1996) e Solanum stramonifolium (Bezerra \& Machado 2003), além de várias espécies de Melastomataceae listadas por Renner (1989). Da mesma maneira, espécies de Augochloropsis, embora geralmente consideradas como abelhas de pequeno porte, são polinizadores efetivos em várias espécies com flores pequenas, como Sauvagesia erecta e S. sprengelii. Essas mesmas abelhas, por outro lado, atuam como pilhadoras ou polinizadoras apenas ocasionais em flores maiores como as de Henriettea succosa, Melastomataceae (Melo \& Machado 1996), e Solanum stramonifolium, Solanaceae (Bezerra \& Machado
2003), que apresentam o dobro ou mais do tamanho das flores das espécies de Sauvagesia aqui estudadas.

A freqüência dos visitantes florais está apresentada na figura 8. As visitas em Sauvagesia erecta iniciam-se em torno de 6:00 h, com maior freqüência entre 7:00 h e 10:00 h da manhã, não sendo mais registradas visitas após as 12:00 h. As espécies de Augochloropsis foram as mais freqüentes, pois visitaram as flores de Sauvagesia erecta durante todo o ano, enquanto as outras espécies só foram observadas em um determinado período do ano. Em S. sprengelii, Florilegus similis foi a espécie mais frequiente, seguida por três espécies de Augochloropsis. As outras duas espécies (Bombus brevivillus e Xylocopa muscaria) foram ocasionais. As visitas começaram às 6:00 h e terminaram às 11:00 h

Tabela 2. Resultados da formação natural de frutos, e das polinizações controladas de Sauvagesia erecta e S. sprengelii.

Table 2. Results of natural fruit set and controlled pollinations of Sauvagesia erecta and S. sprengelii.

\begin{tabular}{|c|c|c|c|c|}
\hline Tratamentos & $\mathrm{N}$ & Frutos formados & Sucesso $(\%)$ & Sementes $(x)$ \\
\hline \multicolumn{5}{|l|}{ Sauvagesia erecta } \\
\hline Apomixia & 10 & 0 & 0 & 0 \\
\hline Autopolinização espontânea & 24 & 9 & 37,50 & $20,2( \pm 10,93)$ \\
\hline Autopolinização manual & 24 & 19 & 79,17 & $17,6( \pm 10,48)$ \\
\hline Polinização cruzada & 17 & 4 & 23,53 & $13,0( \pm 06,16)$ \\
\hline Formação natural de frutos & 26 & 13 & 50,00 & $23,2( \pm 12,64)$ \\
\hline Eficiência do polinizador & 30 & 8 & 26,67 & $26,1( \pm 14,45)$ \\
\hline \multicolumn{5}{|l|}{ Sauvagesia sprengelii } \\
\hline Apomixia & 10 & 0 & 0 & 0 \\
\hline Autopolinização espontânea & 24 & 5 & 20,83 & $3,6( \pm 1,52)$ \\
\hline Autopolinização manual & 18 & 3 & 16,67 & $2,0( \pm 1,00)$ \\
\hline Polinização cruzada & 24 & 4 & 16,70 & $3,8( \pm 4,27)$ \\
\hline Formação natural de frutos & 29 & 18 & 62,07 & $6,4( \pm 2,85)$ \\
\hline
\end{tabular}


Tabela 3. Visitantes florais de Sauvagesia erecta e S. sprengelii. *Segundo terminologia de Wille (1963).

Table 3. Floral visitors of Sauvagesia erecta and S. sprengelii. *According to the terminology of Wille (1963).

\begin{tabular}{|c|c|c|c|}
\hline Visitantes & Comprimento (mm) & Comportamento* & Resultado da visita \\
\hline \multicolumn{4}{|l|}{ Sauvagesia erecta } \\
\hline \multicolumn{4}{|l|}{ Apidae } \\
\hline Paratetrapedia sp.1 & 07 & Vibração & Polinização \\
\hline Paratetrapedia sp. 2 & 07 & Coleta & Pilhagem \\
\hline \multicolumn{4}{|l|}{ Halictidae } \\
\hline Augochloropsis sp.1 & 09 & Vibração & Polinização \\
\hline Augochloropsis sp.2 & 07 & Vibração & Polinização \\
\hline \multicolumn{4}{|l|}{ Sauvagesia sprengelii } \\
\hline \multicolumn{4}{|l|}{ Apidae } \\
\hline Bombus brevivillus Franklin, 1913 & 14 & Vibração & Polinização \\
\hline Florilegus similis Urban, 1970 & 11 & Vibração & Polinização \\
\hline Xуlocoра тизсагіa (Fabrícius, 1779) & 14 & Vibração & Polinização \\
\hline \multicolumn{4}{|l|}{ Halictidae } \\
\hline Augochloropsis sp.1 & 09 & Vibração & Polinização \\
\hline Augochloropsis sp. 3 & 07 & Vibração & Polinização \\
\hline Augochloropsis sp.4 & 06 & Vibração & Polinização \\
\hline
\end{tabular}

da manhã. O período em que se observou o maior número de visitas foi entre 6:00 h e 7:00 h, e entre 7:00 h e 9:00 h houve maior número de espécies visitantes.

A relação da disponibilidade de pólen com a freqüência de visitantes de Sauvagesia erecta e S. sprengelii está representada na figura 9. Logo no início das visitas, há uma queda na quantidade de pólen disponível para os visitantes florais em ambas as espécies, similar àquela que ocorre em Solanum stramonifolium (Bezerra \& Machado 2003). Desse modo, a freqüência de visitas é reduzida em $S$. sprengelii à medida em que o recurso diminui. No entanto, há um aumento no número de visitas em flores de $S$. erecta no período de 8:00 h às 10:00 h, o que pode estar relacionado ao fato de haver um grande número de flores abertas por dia e, na população, muitas ainda não terem sido visitadas. Há uma sensível redução na freqüência de visitantes a partir das 10:00 h (ver figura 8).

Devido ao comportamento das abelhas polinizadoras em ambas espécies, conclui-se que S. erecta e $S$. sprengelii apresentam o mecanismo de polinização por vibração ("buzz-pollination"), descrito por Buchmann (1983). A formação do poro apical pelos estaminódios petalóides provavelmente otimiza a liberação do pólen, direcionando o "jato" de pólen para o corpo do polinizador e minimizando sua perda. Além disso, o cone formado por esses estaminódios impede que algumas abelhas, principalmente aquelas de menor porte, vibrem antera por antera, tornando o contato com o estigma apenas ocasional como ocorre em algumas espécies de Solanum (Symon 1979, Oliveira Filho \& Oliveira 1988, Bezerra \& Machado 2003). Por sua vez, as abelhas vibradoras obrigatoriamente envolvem todo o cone, garantindo o contato com o estigma e possibilitando a polinização. Desse modo, há uma maior seleção de seus polinizadores.

No processo de evolução, a diferenciação das anteras para uma forma tubular alongada e com deiscência poricida ocorreu em resposta ao mecanismo de polinização por vibração (Endress 1994). Anteras poricidas conferem maior proteção ao pólen e diminuem a sua perda, pois direcionam os grãos para o corpo do polinizador, além de limitar o espectro de visitantes florais, visto que só alguns grupos de abelhas são capazes de vibrar as anteras (Endress 1994). Como já discutido, o poro apical formado pelos estaminódios petalóides em S. erecta e $S$. sprengelii desempenha as funções de uma antera poricida. Isso é particularmente interessante no caso das duas espécies de Sauvagesia estudadas, já que ambas apresentam poros com grandes dimensões, quando comparados aos de outras anteras poricidas de famílias distintas, como Solanaceae e Melastomataceae, cujos poros apicais são diminutos. Considera-se, portanto, que em Sauvagesia erecta e S. sprengelii pode ser aplicado o conceito de transferência de função de Corner (1958), embora Kubitzki \& Amaral (1991) só considerem a ocorrência desse fenômeno em representantes de Ochnaceae, quando há a combinação 
A

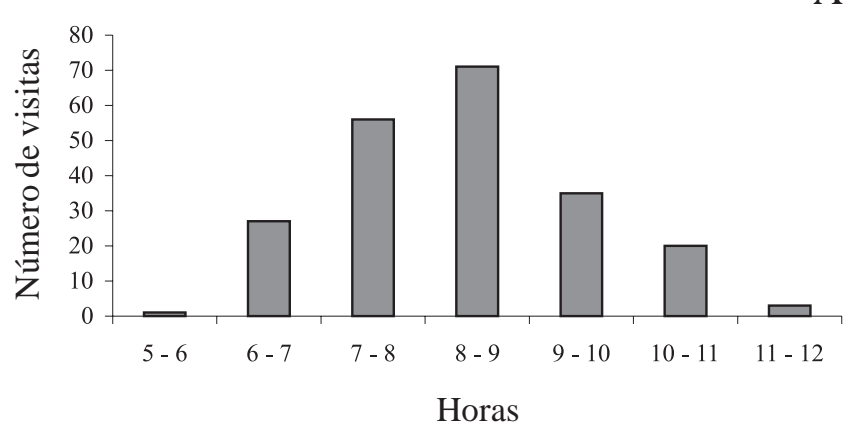

B

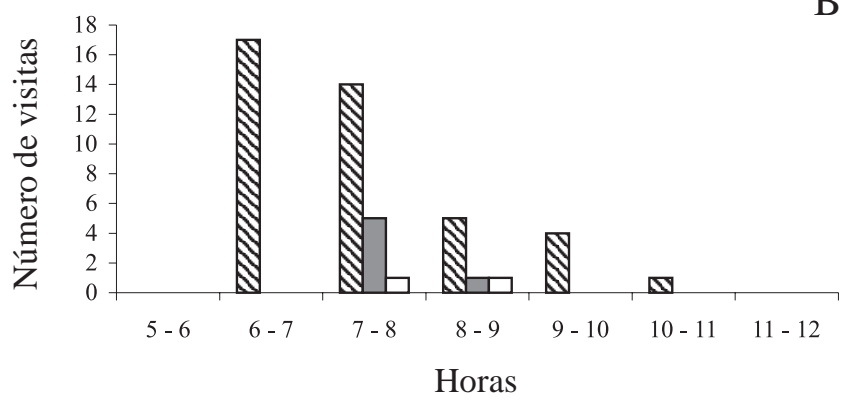

Figura 8. Freqüência de visitas das abelhas em flores de Sauvagesia erecta (A) e Sauvagesia sprengelii (B), durante observações de nove horas consecutivas nos meses de outubro/2001 (para S. erecta) e março/2002 (para S. sprengelii). $\mathbb{\nabla}=$ Florilegus similis; $\square=$ Augochloropsis spp.; $\square=$ outras.

Figure 8. Frequency of visits of the bees at the flowers of Sauvagesia erecta (A) and Sauvagesia sprengelii (B), during observations by nine consecutive hours on the months of October/2001 (to Sauvagesia erecta) and March/2002 (to $S$. sprengelii). $\mathbb{\nabla}=$ Florilegus similis; $\square=$ =Augochloropsis spp.; $\square=$ others.

do cone de estaminódios com anteras de deiscência longitudinal.

Agradecimentos - À Ana Paula Miranda (UFPE) e à MSc. Ana Virgínia Leite (UFPE) pela ajuda nas atividades de campo; Drs. Ariadna Lopes (UFPE) pelas sugestões ao manuscrito e correção do Abstract, Roxana Barreto (UFPE) e Rita de Cássia Pereira (IPA/PE) pela identificação das espécies vegetais, Maria do Carmo Amaral (Unicamp) pela confirmação da identificação de S. erecta, Isabel Alves dos Santos (Unesc), Gabriel Melo (UFPR) e Danuncia Urban (UFPR) pela gentileza na identificação das espécies de abelhas; à Diretoria do Parque Estadual Dois Irmãos pela autorização da pesquisa no campo; aos dois assessores anônimos, pelas valiosas sugestões ao manuscrito; ao PIBIC/UFPE/CNPq pela bolsa de iniciação científica e ao CNPq pela bolsa de Produtividade em Pesquisa, respectivamente para as primeira e segunda autoras.
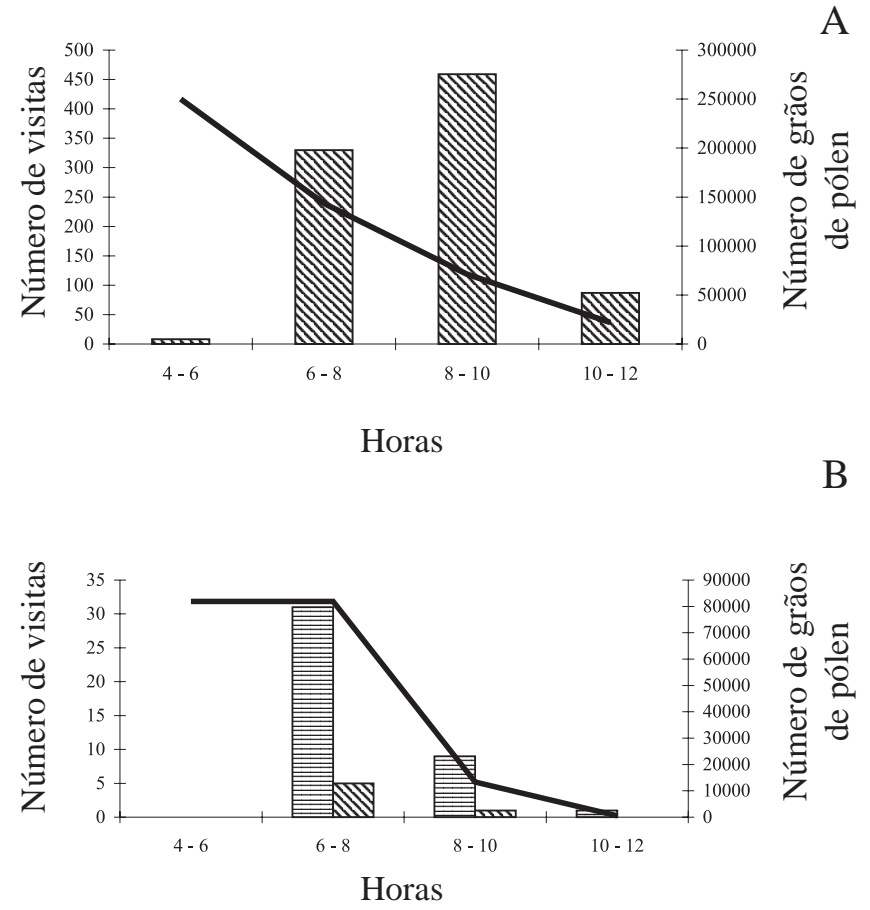

Figura 9. Relação da disponibilidade de pólen com a frequiência de visitantes de Sauvagesia erecta (A) e Sauvagesia sprengelii (B). 目 = Florilegus similis; $\mathbb{Q}=$ Augochloropsis spp.; $-=$ número de grãos de pólen.

Figure 9. Relation between pollen availability and frequency of visitors to Sauvagesia erecta (A) and Sauvagesia sprengelii $(\mathrm{B})$. 目 $=$ Florilegus similis $; \mathbb{\mathbb { Q }}=$ Augochloropsis spp.; $-=$ number of pollen grains.

\section{Referências bibliográficas}

AMARAL, M.C.E. \& BITTRICH, V. 1998. Ontogenia inicial do androceu de espécies de Ochnaceae subfam. Sauvagesioideae através da análise em microscopia eletrônica de varredura. Revista Brasileira de Botânica 21:269-273.

AYRES, M., AYRES, J.R.M., AYRES, D.L. \& SANTOS, A.S. 2000. BioEstat 2.0: Aplicações estatísticas nas áreas de ciências biológicas e médicas. Sociedade Civil Mamirauá/ CNPq, Belém.

BARROSO, G. 1978. Sistemática de Angiospermas do Brasil. v.1. Livros Técnicos e Científicos. Universidade de São Paulo, Rio de Janeiro/ São Paulo.

BENCKE, C.S.C. \& MORELLATO, L.P.C. 2002. Comparação de dois métodos de avaliação da fenologia de plantas, sua interpretação e representação. Revista Brasileira de Botânica 25:269-275.

BEZERRA, E.L.S. \& MACHADO, I.C. 2003. Biologia floral e sistema de polinização de Solanum stramonifolium Jacq. (Solanaceae) em remanescente de Mata Atlântica Pernambuco. Acta Botanica Brasilica 17:247-257. 
BUCHMANN, S.L. 1983. Buzz pollination in angiosperms. In Handbook of Experimental Pollination (C.E. Jones \& R.J. Little, eds.). Van Nostrand Reinhold, New York, p.73-113.

BUCHMANN, S.L., JONES, C.E. \& COLIN, L.J. 1978. Vibratile pollination of Solanum douglasii and S. xanti (Solanaceae) in Southern California. The Wasmann Journal of Biology 35:1-25.

CORNER, E.J.H. 1958. Transference of function. The Journal of the Linnean Society 56:33-40.

COUTINHO, R.Q., LIMA FILHO, M.F., SOUZANETO, J.B. \& SILVA, E.P. 1998. Características climáticas, geológicas, geomorfológicas e geotécnicas da Reserva Ecológica de Dois Irmãos. In Reserva Ecológica de Dois Irmãos: Estudos em um Remanescente de Mata Atlântica em Área Urbana (Recife - Pernambuco - Brasil) (I.C. Machado, A.V. Lopes \& K.C. Porto, orgs.). Secretaria de Ciência, Tecnologia e Meio Ambiente / Universidade Federal de Pernambuco, Recife, p.21-49.

CRUDEN, R.W. 1977. Pollen-ovule ratios: A conservative indicator of breeding systems in flowering plants. Evolution 31:32-46.

CRUDEN, R.W. 2000. Pollen grains: why so many? Plant Systematics and Evolution 222:143-165.

ENDRESS, P.K. 1994. Diversity and evolutionary biology of tropical flowers. Cambridge University Press, Cambridge.

GENTRY, A.H. 1974. Flowering phenology and diversity in tropical Bignoniaceae. Biotropica 6:64-68.

GOTTSBERGER, G. \& SILBERBAUER-GOTTSBERGER, I. 1988. Evolution of flower structures and pollination in neotropical Cassiinae (Caesalpiniaceae) species. Phyton 28:293-320.

HENRIQUES, R.P.B. 1999. Ecologia da polinização de Ouratea hexasperma (St. Hil.) Baill. (Ochnaceae) em cerrado no Brasil Central. Boletim do Herbário Ezechias Paulo Heringer 4:46-64.

HOUSTON, T.F. \& LADD, P.G. 2002. Buzz pollination in the Epacridaceae. Australian Journal of Botany 50:83-91.

KUBITZKI, K. \& AMARAL, M.C.E. 1991. Transference of function in the pollination system of the Ochnaceae. Plant Systematics and Evolution 177:77-80.

MAÊEA, J.M. 1985. Manual para uso da câmara de Newbauer para contagem de pólen em espécies florestais. Universidade Federal Rural do Rio de Janeiro, Departamento de Silvicultura, Seropédica.

MARTINS, C.F. 1995. Flora apícola e nichos tróficos de abelhas (Hym., Apoidea) na Chapada Diamantina (Lençóis-BA, Brasil). Revista Nordestina de Biologia 10:119-140.

MELO, G.F.A. \& MACHADO, I.C. 1996. Biologia da reprodução de Henriettea succosa DC. (Melastomataceae). Revista Brasileira de Biologia 56:383-389.

MELO, G.F.A. \& MACHADO, I.C. 1998. Auto-incompatibilidade em Miconia ciliata (L.C. Rich.) DC. (Miconieae Melastomataceae). Acta Botanica Brasilica 12:113-120.

MICHENER, C.D., WINSTON, M.L. \& JANDER, R. 1978. Pollen manipulation and related activities and structures in bees of the family Apidae. The University of Kansas Science Bulletin 51:575-601.
NEWSTROM, L.E., FRANKIE, G.W. \& BAKER, H.G. 1994. A new classification for plant phenology based on flowering patterns in lowland Tropical Rain Forest Trees at La Selva, Costa Rica. Biotropica 26:141-159.

OLIVEIRA FILHO, A.T. \& OLIVEIRA, L.C.A. 1988. Biologia floral de uma população de Solanum lycocarpum St. Hil. (Solanaceae) em Lavras, MG. Revista Brasileira de Botânica 11:23-32.

PINHEIRO, M.C.B., LIMA, H.A., ORMOND, W.T. \& CORREIA, M.C.R. 1999. Ouratea cuspidata (St. Hil.) Engler (Ochnaceae): um caso especial de antese. Boletim do Museu Nacional, nova série, Botânica. 16:1-11.

PRIMACK, R.B. 1985. Longevity of individual flowers. Annual Review of Ecology and Systematics 16:15-37.

RADFORD, A.E., DICKINSON, W.C., MASSEY, J.R. \& BELL, C.R. 1974. Vascular plant systematics. Harper \& Row, New York.

RENNER S. 1983. The widespread occurrence of anther destruction by Trigona bees in Melastomataceae. Biotropica 15:251-256.

RENNER S. 1989. A survey of reproductive biology in neotropical Melastomataceae and Memecylaceae. Annals of Missouri Botanical Garden 76:496-518.

RICHARDS, A.J. 1986. Plant Breeding Systems. Unwin \& Allem, London.

ROBINSOHN, I. 1924. Die farbungsreaktion der narbe, stigmatochromie, als morpho-biologische Blütenuntersuchungsmethode. Sitzungsberichten Akademischer Wissenschaft Wien, Mathematics, Abteilung 133:180-213.

STEBBINS, G.L. 1957. Self fertilization and population variability in the higher plants. The American Naturalist 91:337-354.

STRATTON, D.A. 1989. Longevity of individual flowers in a Costa Rican cloud forest: ecological correlates and phylogenetic constraints. Biotropica 21:308-318.

SYMON, D.E. 1979. Sex forms in Solanum (Solanaceae) and the role of pollen collecting insects. In The Biology and Taxonomy of the Solanaceae (J.G. Hawkes, R.N. Lester \& A.D. Skelding, eds.). Academic Press, London, p.385-398.

THORP, R.W. \& ESTES, J.R. 1975. Intrafloral behavior of bees on flowers of Cassia fasciculata. Journal of the Kansas Entomological Society 48:175-184.

VOGEL, S. 1978. Evolutionary shifts from reward to deception in pollen flowers. In The pollination of flowers by insects (A.J. Richards, ed.). Academic Press, London, p.89-96.

WENDT, T., CANELA, M.B.F., KLEIN, D.E. \& RIOS, R.I. 2002. Selfing facilitates reproductive isolation among three sympatric species of Pitcairnia (Bromeliaceae). Plant Systematics and Evolution 232:201-212.

WILLE, A. 1963. Behavioral adaptations of bees for pollen collecting from Cassia flowers. Revista de Biología Tropical 11:205-210. 